\title{
BIBLIOGRAPHY
} 520.

1. A. A. Albert, Quasigroups. I, Trans. Amer. Math. Soc. vol. 54 (1943) pp. 507-

2. R. Baer, The homomorphism theorems for loops, Amer. J. Math. vol. 67 (1945) pp. $450-460$.

3. R. H. Bruck, Simple quasigroups, Bull. Amer. Math. Soc. vol. 50 (1944) pp. 769-781.

4. - Some results in the theory of linear non-associative algebras, Trans. Amer. Math. Soc. vol. 56 (1944) pp. 141-199.

5. G. H. Garrison, Quasigroups, Ann. of Math. vol. 41 (1940) pp. 474-487.

6. F. Kiokemeister, $A$ theory of normality for quasigroups, Amer. J. Math. vol. 70 (1948) pp. 99-106.

7. B. L. van der Waerden, Moderne Algebra, Berlin, 1930, 1st ed.

MT. Holyoke College

\section{A CONJECTURE OF KRISHNASWAMI}

\section{H. LEHMER}

Let $T(N)$ denote the number of right triangles whose perimeters do not exceed $2 N$, and whose sides are relatively prime integers. A list of all such triangles whose perimeters do not exceed 10000 has been given by A. A. Krishnaswami. ${ }^{1}$ On the basis of this table he conjectured that

$$
T(N) \sim N / 7 .
$$

The asymptotic formula

$$
T(N) \sim \pi^{-2} N \log 4
$$

follows from the general theory of "totient points," as developed by D. N. Lehmer in 1900. A statement equivalent to (2) will be found in his $\operatorname{paper}^{2}$ (p. 328).

The conjecture (1) is not far wrong since

$$
\pi^{2} / \log 4=7.11941466 \text {. }
$$

Presented to the Society, April 17, 1948; received by the editors January 29, 1948.

${ }^{1}$ A. A. Krishnaswami, On isoperimetrical Pythagorean triangles, Tôhoku Math. J. vol. 27 (1926) pp. 332-348. Two omissions in Table I may be noted: For $s=3450$, $a=50, b=19$; for $s=3465, a=55, b=8$. This table is the basis for the one at the end of the present paper.

${ }^{2}$ D. N. Lehmer, Asymptotic evaluation of certain totient sums, Amer. J. Math. vol. 22 (1900) pp. 293-335. 
In this paper we give a short proof of the fact that

$$
T(N)=\pi^{-2} N \log 4+O\left(N^{1 / 2} \log N\right) .
$$

The actual values of the error term for $N=500(500) 5000$ are given in a short table at the end of this paper. The proof of (3) is based on the following lemmas.

Lemma 1. Let $\phi(m)$ denote the number of positive integers not exceeding $m$ and prime to $m$. Then

$$
\Phi(x) \equiv \sum_{m \leqq x} \phi(m)=3 \pi^{-2} x^{2}+O(x \log x) .
$$

A proof of this well known result will be found for example in Hardy and Wright ${ }^{8}$ (p. 266).

Lemma 2. Let $\Phi_{\odot}(x)$ and $\Phi_{0}(x)$ be defined by

$$
\Phi_{e}(x)=\sum_{m \geqq x, m \text { even }} \phi(m), \quad \Phi_{0}(x)=\sum_{m \leqq x, m \text { odd }} \phi(m) .
$$

Then

$$
\begin{aligned}
& \Phi_{e}(x)=\pi^{-2} x^{2}+O(x \log x), \\
& \Phi_{0}(x)=2 \pi^{-2} x^{2}+O(x \log x) .
\end{aligned}
$$

Proof. Since (6) follows from (4) and (5) it suffices to prove (5). To this effect we note that if $m$ is even

$$
\phi(m)=\left\{\begin{array}{cc}
\phi(m / 2), & m \equiv 2(\bmod 4), \\
2 \phi(m / 2), & m \equiv 0(\bmod 4) .
\end{array}\right.
$$

Hence

$$
\Phi_{0}(x)=\Phi_{0}(x / 2)+2 \Phi_{\theta}(x / 2)=\Phi(x / 2)+\Phi_{0}(x / 2) .
$$

Therefore

$$
\Phi_{c}(x)=\sum_{\lambda=1}^{p} \Phi\left(2^{-\lambda} x\right) \quad(p=[\log x / \log 2]) .
$$

Applying Lemma 1 we have

$$
\Phi_{e}(x)=3 \pi^{-2} x^{2} \sum_{\lambda=1}^{p} 4^{-\lambda}+O(x \log x)
$$

${ }^{3}$ G. H. Hardy and E. M. Wright, Introduction to the theory of numbers, Oxford, 1938. Lemma 1 appears to be due to Mertens, Journal für Mathematik vol. 77 (1871) pp. 289-291. 


$$
=\pi^{-2} x^{2}+O\left(x^{2} \int_{p}^{\infty} 4^{-t} d t\right)+O(x \log x)
$$

Since $p>\log x / \log 4$, the integral is $O(1 / x)$. Hence (5) follows.

Lemma 3. Let $0<\theta<1$, and define $F(\theta, x), F_{e}(\theta, x)$ and $F_{0}(\theta, x)$ by

$$
\begin{gathered}
F(\theta, x)=\sum_{\theta x<m \leqq x} m^{-2} \phi(m), \quad F_{e}(\theta, x)=\sum_{\theta x<m \leqq x, m \text { even }} m^{-2} \phi(m), \\
F_{0}(\theta, x)=\sum_{\theta x<m \leqq x, m \text { odd }} m^{-2} \phi(m) .
\end{gathered}
$$

Then as $x \rightarrow \infty$, with $\theta$ fixed,

$$
\begin{aligned}
& F(\theta, x)=-6 \pi^{-2} \log \theta+O\left(x^{-1} \log x\right), \\
& F_{e}(\theta, x)=-2 \pi^{-2} \log \theta+O\left(x^{-1} \log x\right), \\
& F_{0}(\theta, x)=-4 \pi^{-2} \log \theta+O\left(x^{-1} \log x\right) .
\end{aligned}
$$

Proof. Since (10) follows from (8) and (9) it suffices to prove (8) and (9). Now

$$
\begin{aligned}
F(\theta, x)= & \sum_{\theta x<m \leqq x} m^{-2} \phi(m)=\sum_{\theta x<m \leqq x}\{\Phi(m)-\Phi(m-1)\} m^{-2} \\
= & \sum_{\theta x<m \leqq x} \Phi(m)\left\{m^{-2}-(m+1)^{-2}\right\} \\
& -\Phi(\theta x)[\theta x+1]^{-2}+\Phi(x)[x+1]^{-2} .
\end{aligned}
$$

By Lemma 1 these last two terms cancel to some extent and together contribute only $O\left(x^{-1} \log x\right)$. As for the rest

$$
\begin{aligned}
& \sum_{\theta x<m \leqq x} \Phi(m)\left\{m^{-2}-(m+1)^{-2}\right\} \\
& \quad=3 \pi^{-2} \sum\left(1-\left(1+m^{-1}\right)^{-2}\right)+O\left(\sum m^{-1}\left(1-\left(1+m^{-1}\right)^{-2}\right) \log m\right) \\
& \quad=3 \pi^{-2} \sum 2 m^{-1}\left(1+O\left(m^{-1}\right)\right)+O\left(\sum m^{-2} \log m\right) \\
& \quad=6 \pi^{-2} \int_{\theta x}^{x} t^{-1} d t+O\left(x^{-1}\right)+O\left(\int_{\theta x}^{x} t^{-2} \log t d t\right) \\
& =-6 \pi^{-2} \log \theta+O\left(x^{-1} \log x\right),
\end{aligned}
$$

which gives (8). To prove (9) we note from (7) that

$$
F_{\circ}(\theta, x)=F_{0}(\theta, x / 2) / 4+F_{\odot}(\theta, x / 2) / 2=F(\theta, x / 2) / 4+F_{0}(\theta, x / 2) / 4 \text {. }
$$

Hence

$$
F_{e}(\theta, x)=\sum_{\lambda=1}^{p} F\left(\theta, x / 2^{\lambda}\right) 4^{-\lambda} \quad(p=[\log x / \log 2]) .
$$


Using (8) we find

$$
F_{e}(\theta, x)=-6 \pi^{-2} \log \theta \sum_{\lambda=1}^{\infty} 4^{-\lambda}+O\left(\int_{p}^{\infty} 4^{-t} d t\right)+O\left(x^{-1} \log x\right) .
$$

Since the integral is $O\left(x^{-1}\right),(9)$ follows at once. This completes the proof of Lemma 3.

Lemma 4. Let $\phi(x, m)$ denote the number of integers $\leqq x$ and prime to $m$. Then

$$
\left|\phi(x, m)-x m^{-1} \phi(m)\right|<d(m)
$$

where $d(m)$ is the number of divisors of $m$.

This follows easily from a familiar theorem of Legendre to the effect that

$$
\phi(x, m)=\sum_{\delta \mid m}\left[x \delta^{-1}\right] \mu(\delta)
$$

where $\mu$ is the Möbius function and the sum extends over all the divisors of $m$. In fact if we write

$$
\left[x \delta^{-1}\right]=x \delta^{-1}-\epsilon(x, \delta)
$$

so that

$$
0 \leqq \epsilon(x, \delta)<1,
$$

then (11) becomes

$$
\phi(x, m)=x \sum \delta^{-1} \mu(\delta)-\sum \epsilon(x, \delta) \mu(\delta) .
$$

The first sum is $m^{-1} \phi(m)$ and the second is less than

$$
\sum_{\delta \mid m} 1=d(m)
$$

in absolute value. This proves the lemma.

Finally we need one more lemma.

LEMMA. 5.

$$
\sum_{m \leqq x} d(m)=O(x \log x)
$$

This is a very weak corollary of a famous result of Dirichlet (see Hardy and Wright, ${ }^{3}$ p. 262-263).

We are now in a position to prove the following theorem.

ThEOREM. Let $T(N)$ denote the number of integral right triangles whose perimeters do not exceed $2 N$ and whose sides are relatively prime, then 


$$
T(N)=\pi^{-2} N \log 4+O\left(N^{1 / 2} \log N\right) .
$$

Proof. It is well known that all integral right triangles $(a, b, c)$ are given by the parametric equations

$$
a=m^{2}-n^{2}, \quad b=2 m n, \quad c=m^{2}+n^{2}
$$

where $m, n$ are integers with

$$
n \leqq m \text {. }
$$

Since the perimeter is supposed not to exceed $2 N$ we have

$$
m n+m^{2} \leqq N .
$$

In order to avoid the cases in which $a, b, c$ have a common factor it is necessary to suppose that we choose $m, n$ so that

$$
m, n \text { are coprime and not both odd. }
$$

$T(N)$ is then merely the number of pairs of positive integers $(m, n)$ such that (12), (13) and (14) hold. In case $m \leqq(N / 2)^{1 / 2},(13)$ is a consequence of (12). In case $(N / 2)^{1 / 2}<m \leqq N^{1 / 2}$, (12) is a consequence of (13). Hence if we define

$$
\psi(m)=\left\{\begin{array}{cll}
1 & \text { if } & m \leqq(N / 2)^{1 / 2} \\
m^{-2} N-1 & \text { if } & (N / 2)^{1 / 2}<m \leqq N^{1 / 2}
\end{array}\right.
$$

then the number of integers $n$ that go with a given $m$ is the number of integers prime to $m$ not exceeding $m \psi(m)$ or $m \psi(m) / 2$ according as $m$ is even or odd. Hence if we set $x=N^{1 / 2}$

$$
T(N)=\sum_{m \leqq x, m \text { even }} \phi(m \psi(m), m)+\sum_{m \leqq x, m \text { odd }} \phi\left(\frac{m \psi(m)}{2}, m\right) .
$$

By Lemma 4,

$$
\begin{aligned}
T(N) & =\sum_{m \leqq x, m \text { even }} \psi(m) \phi(m)+2^{-1} \sum_{m \leqq x, m \text { odd }} \psi(m) \phi(m)+R(N) \\
& =\sum_{1}+2^{-1} \sum_{2}+R(N)
\end{aligned}
$$

where

$$
|R(N)| \leqq \sum_{m \leqq x} d(m)=O(x \log x)=O\left(N^{1 / 2} \log N\right) .
$$

By (15) with $\theta=2^{-1 / 2}$ we can write

$$
\sum_{1}=\Phi_{e}(\theta x)+N F_{e}(\theta, x)-\Phi_{e}(x)+\Phi_{e}(\theta x),
$$




$$
\sum_{2}=\Phi_{0}(\theta x)+N F_{0}(\theta, x)-\Phi_{0}(x)+\Phi_{0}(\theta x)
$$

so that

$$
\begin{aligned}
\sum_{1}+2^{-1} \sum_{2}= & \Phi(\theta x)+\Phi_{e}(\theta x)-2^{-1}\left\{\Phi(x)+\Phi_{e}(x)\right\} \\
& +2^{-1} N\left\{F(\theta, x)+F_{e}(\theta, x)\right\} .
\end{aligned}
$$

By Lemmas 1, 2, and 3 therefore we obtain after simplification

$T(N)=\pi^{-2}(2 \log 2) x^{2}+O(x \log x)=\pi^{-2}(\log 4) N+O\left(N^{1 / 2} \log N\right)$.

The following small table illustrates the error in (3):

$$
E(N)=T(N)-\pi^{-2} N \log 4 .
$$

The function $C(N)$ is defined by

$$
C(N) N^{1 / 2} \log N=10^{3} E(N)
$$

and gives some idea of the possible constant implied by the $O$ term of (3).

$\begin{array}{rccrcc}N & T(N) & \Delta T & \pi^{-2} N \log 4 & E(N) & C(N) \\ 500 & 70 & 70 & 70.23049 & -0.23049 & -1.6596 \\ 1000 & 140 & 71 & 140.46099 & -0.46099 & -2.1103 \\ 1500 & 211 & 69 & 210.69148 & +0.30852 & +1.0893 \\ 2000 & 280 & 69 & 280.92197 & -0.92197 & -2.7123 \\ 2500 & 349 & 73 & 351.15246 & -2.15246 & -5.5022 \\ 3000 & 422 & 70 & 421.38296 & -0.61704 & -1.4071 \\ 3500 & 492 & 68 & 491.61345 & +0.38655 & +0.8007 \\ 4000 & 560 & 71 & 561.84394 & -1.84394 & -3.5152 \\ 4500 & 631 & 72 & 632.07444 & -1.07444 & -1.9041 \\ 5000 & 703 & & 702.30493 & +0.69507 & +1.1541\end{array}$

University of CALIFornia 